Published in final edited form as:

IEEE Nucl Sci Symp Conf Rec (1997). 2008 October ; 11(2008): 4494-4501. doi:10.1109/NSSMIC.

2008.4774291.

\title{
Clinical Imaging Characteristics of the Positron Emission Mammography PEM Flex Solo II
}

\author{
Lawrence MacDonald [Member IEEE], \\ Radiology Dept., Univ. of Washington, Seattle, WA, 98195 \\ John Edwards, \\ Providence Centralia Hospital, Centralia, WA 98531 \\ Thomas Lewellen [Fellow IEEE], \\ Radiology Dept., Univ. of Washington, Seattle, WA, 98195 \\ James Rogers, and \\ Swedish Cancer Institute, Seattle, WA 98104 \\ Paul Kinahan [Senior Member IEEE] \\ Radiology Dept., Univ. of Washington, Seattle, WA, 98195
}

\section{Abstract}

Positron emission mammography (PEM) uses two opposing gamma-ray imagers and limited-angle tomography techniques to image radiotracer distributions within the breast. Due to their smaller size and closer proximity to the source, dedicated PEM cameras can provide better spatial resolution and count sensitivity than whole-body positron emission tomographs. We performed several clinical imaging tests on a commercially available PEM camera, the PEM Flex Solo II. This system is comprised of two opposing $6 \mathrm{~cm} \times 16.4 \mathrm{~cm}$ detectors that scan in unison to cover up to a $24 \mathrm{~cm} \times$ $16.4 \mathrm{~cm}$ field of view (FOV). We measured spatial resolution, uniformity, recovery coefficients (RC), and quantification using the system clinical software. Image linearity and coefficient of variation $(\mathrm{CV})$ at the edge of the FOV were also characterized. Anecdotal examples of clinical patient data are presented. Spatial resolution is $2.4 \mathrm{~mm}$ FWHM for image planes parallel to the detector faces; background variability is 6\%; quantification and $\mathrm{RC}$ varied within the FOV; positioning linearity began at $\sim 13 \mathrm{~mm}$ from the edge of the detector housing; CV increased rapidly at the edge of the FOV due to limited sampling in these image planes.

\section{Introduction}

Positron emission mammography (PEM) is an imaging technique dedicated to measuring the distribution of positron-emitting radiotracers in the breast. PEM, like other nuclear medicine techniques, provides functional, or molecular imaging information. This is in contrast to x-ray mammography, ultrasound (US), and magnetic resonance imaging (MRI), which primarily provide anatomical or structural features of the tissue. PEM thus provides additional, complementary information to the conventional breast imaging modalities. X-ray mammography is believed to be responsible for reversing a trend of increased breast cancer deaths that existed prior to regular mammography screening [1]. However, despite the successes of earlier detection by screening mammography, breast cancer is the second-leading 
cause of cancer-related deaths in North American women (following lung cancer mortality). This statistic shows a clear need for improved methods to fight breast cancer.

Whole-body positron emission tomography (WB-PET) has been used for imaging breast cancer patients [2]. WB-PET systems typically have intrinsic spatial resolution of $\sim 5-7 \mathrm{~mm}$ full width at half maximum (FWHM), and in practice, reconstructed resolution is 10-15 mm FWHM. As a result, breast lesions imaged using WB-PET are associated with relatively advanced disease. Ideally, cancer is treated in the very early stages of the disease. The idea of dedicated PEM systems is to reduce the size threshold of functional breast imaging, and thus allow earlier intervention.

PEM was proposed in the 1990s [3], and has continued to receive attention from the research community since then [4]. Naviscan Inc. now offers a commercial PEM scanner. The PEM Flex Solo II is the latest generation PEM scanner offered by Naviscan.

Dedicated breast PET and PEM systems are still in early stages of development and implementation. No camera testing standards have been developed for these systems. As is the case with small animal PET scanners, the conventional WB-PET testing standards are inappropriate for characterizing dedicated breast imaging systems because of their distinct design and application. In this work we tested several common imaging parameters using the standard clinical operating mode of the PEM Flex system; spatial resolution, uniformity, and recovery coefficients were measured. In addition, we investigated properties relevant to the breast imaging application, namely imaging properties of the PEM Flex at the edge of the field of view (FOV), which is important for imaging posterior breast lesions.

In a separate, independent study, the PEM Flex Solo II was characterized using the NEMA NU 4-2008 Small Animal PET Standard and results were presented at the 2008 IEEE NSSMIC conference in Dresden, Germany [5].

\section{Materials and Methods}

\section{A. PEM Flex Solo II System}

The PEM Flex Solo II has two opposing gamma ray imaging detectors mounted inside compression paddles used to immobilize and mildly compress the breast. A photograph of the system is shown in figure 1 . The gamma ray detectors are $\sim 6 \mathrm{~cm} \times 16.4 \mathrm{~cm}$ in imaging area, and $\sim 6 \mathrm{~cm}$ thick. The detectors scan across the FOV in the direction of their $6 \mathrm{~cm}$ dimension to cover up to $24 \mathrm{~cm}$, making the full FOV of the system $24 \mathrm{~cm} \times 16.4 \mathrm{~cm}$. The detectors are constructed from $2 \mathrm{~mm} \times 2 \mathrm{~mm} \times 13 \mathrm{~mm}$ LYSO scintillation crystals coupled to positionsensitive photomultiplier tubes.

The detectors are mounted on an articulating arm that rotates to allow imaging the breast from different views (figure 1). Acquisition options on the PEM Flex Solo II at the Swedish Cancer Institute include the scan range FOV (up to $24 \mathrm{~cm}$ ), and the acquisition duration. The image reconstruction uses a 3D list-mode maximum-likelihood expectation maximization (MLEM) algorithm. No corrections are made for scattered or accidental coincidence detections, and there are no user-selectable reconstruction filters or other options.

The PEM Flex Solo II is a limited-angle tomography system. Limited-angle tomography is also referred to as tomosynthesis. In this technique, image planes parallel to the detectors ('inplane' images) are well sampled and reconstructed with a spatial resolution limited by the intrinsic sampling of the detectors. However, image planes perpendicular to the detector faces ('cross-planes') lack sufficient sampling to reconstruct at the same high-resolution integrity. While tomosynthesis suffers from anisotropic spatial resolution, and other 3D inaccuracies, it 
does provide improved volumetric imaging over single-view planar projection imaging. Due to the anisotropy, two imaging views are required in order to achieve high-resolution imaging in all three dimensions. The PEM Flex Solo II system generates in-plane images with $1.2 \mathrm{~mm}$ pixel size, and 12 or 24 in-plane slices. As such, the cross-plane pixel sizes are variable, determined by the detector separation (compression thickness) divided by 12 or 24 .

The PEM Flex Solo II reports image values in either absolute activity concentration (e.g. $\mu \mathrm{Ci} /$ cc), or using a proprietary form of the standardized uptake value (SUV). All of the results in this paper were derived from the proprietary SUV obtained from DICOM images. We note that some variation was seen in DICOM image values depending on the software used - we are investigating the cause of the variation.

\section{B. Detector Characterization}

i) Spatial Resolution-We measured spatial resolution by imaging $1.0 \mathrm{~mm}$ inner diameter capillary tubes that contained a small amount of $18-\mathrm{F}$ solution. One or more such capillary tubes were imaged at different locations within the FOV, and with compression distances of 3 $\mathrm{cm}, 6 \mathrm{~cm}$, and $9 \mathrm{~cm}$. Spatial resolution was calculated on the image slice with maximum counts by fitting a profile of the line source to a Gaussian curve. Profiles of the line source were always taken perpendicular to the length of the capillary tube. We point out that this determination of spatial resolution deviates from the conventional method of determining spatial resolution in a PET scanner as prescribed by NEMA standards in at least three ways; it is recommended that spatial resolution be calculated from an image reconstructed using an analytical filtered backprojection method, whereas here we used the MLEM reconstruction available to us on the clinical PEM Flex system; secondly, the resolution we measured was limited by the image pixel size; third, FWHM is not determined from a Gaussian fit in the NEMA standard [6].

ii) Uniformity-A uniform source was made by injecting $2.6 \mathrm{MBq}(70 \mu \mathrm{Ci})$ of $18-\mathrm{F}$ solution into a $500 \mathrm{cc}$ intravenous (IV) saline bag. This activity concentration corresponds to $370 \mathrm{MBq}$ $(10 \mathrm{mCi})$ distributed uniformly in 70 liters of water. Two such sources were prepared, stacked together, and compressed in the PEM Flex FOV such that a portion of the activity distribution was outside of the FOV. The source was positioned in this way to measure imaging effects at the edge of the FOV. Air was removed from the IV bags, and they were compressed between the detectors with a $55 \mathrm{~mm}$ compression thickness. A $18 \mathrm{~cm}$ wide scan FOV was used to image the $11.8 \mathrm{~cm}$ wide source for $1,3,7$, and 15 minutes.

Profiles through the uniform source in each image dimension were graphed for visual assessment of uniformity. The background variability was calculated using the NEMA standard modified as deemed appropriate for this source. We modified the NEMA specification by using $2.0 \mathrm{~cm}$ diameter regions of interest (ROIs), six such ROls were drawn on each of the 24 inplane image slices, and the background variability was calculated as specified in [6] using these 144 ROls.

iii) Edge Effects-PET and PEM scanners have minimum coincidence detection sensitivity at the edge-most detector rings of the system. For a PEM system this can be thought of as the cross-planes at either edge of the detector pair. For WB-PET the effect of low sensitivity at the edge of the FOV is overcome by scanning additional axial positions, so that the desired anatomy of the patient is in the center of the FOV, and by overlapping adjacent axial acquisitions to help mitigate sensitivity variability. This approach is not possible with a PEM system because the torso of the patient prevents positioning the detectors such that the posterior breast is in the center of the FOV. As such, the posterior portion of the PEM image, i.e. at the chest-wall of the patient, will be imaged with lower counting statistics than regions closer to the FOV center. 
The lower counting statistics lead to an inevitable increase in image variance in those image planes.

Two image properties were studied at the edge of the PEM Flex FOV: the positioning limits and linearity, and the coefficient of variation $(\mathrm{CV})$ of image planes and profiles. The purpose of the linearity measurement was to determine the distance between the accurate imaging field and the physical edge of the detector. A line source was imaged near the detector edge to measure the limits and linearity. The CV was calculated from both 2-D ROIs (7 cm wide X the full compression distance in cross-planes), and I-D profiles ( $7 \mathrm{~cm}$ long) near the FOV edge. Only in-plane images are viewed in practice, and the I-D CV represents variance observed on these images. The 2-D CV averages the effect over cross-planes. CV was calculated on the uniformity images described above.

iv) Recovery Coefficients-We used plastic spheres filled with an activity concentration four times (4X) that of the background to measure recovery coefficients (RC). The IV bags described above in the uniformity measurement served as the background activity. The spheres (the Standard and Micro Hollow Sphere Sets, Data Spectrum Corp., Hillsborough, NC) were imaged two at a time close to the center of the in-plane FOV. This was repeated with the spheres between the two IV bags, and below the two IV bags in contact with one of the detectors. The background concentration was approximately $5 \mathrm{kBq} / \mathrm{ml}(0.14 \mu \mathrm{Ci} / \mathrm{ml})$. Sphere inner diameters ranged from $4 \mathrm{~mm}$ to $31 \mathrm{~mm}$.

It is difficult or impossible to draw ROIs within small source structures in a way that excludes surrounding background image pixels. Consequently, on patient images at the Swedish Cancer Institute, ROIs larger than the sources of interest are drawn around the sources, and the image pixel maxima in the ROIs are recorded as the measure of lesion uptake. Large ROIs are also drawn around background areas of the image, away from the source of interest, and the image pixel mean of these ROIs is taken as the measure of background uptake. Lesion-to-background ratios are calculated with these two measurements. This methodology was followed for the calculation ofthe $\mathrm{RC}$ in this work.

In addition to measuring $\mathrm{RC}$ as a function of sphere size, we measured the relative quantification of a single source placed at four cardinal locations within the FOV of the PEM Flex system. We used the $2 \mathrm{~cm}$ diameter sphere for this, and placed it in the four positions shown in figure 2.

We made the RC and quantification measurements from ROIs drawn on the in-plane image slice with the highest mean ROI value, as that was the quantity that varied uniformly across all in-plane slices.

v) Clinical Protocol-At the Swedish Cancer Institute the PEM Flex Solo II is used to image patients that have already been diagnosed with breast cancer and are receiving a WB-PET/CT scan. The WB-PET/CT protocol includes patient fasting for 6-12 hours, requiring blood glucose levels less than $150 \mathrm{mg} / \mathrm{dl}$, injecting $600 \mathrm{MBq}(16 \mathrm{mCi})$ of FDG, $60 \mathrm{~min}$. uptake time, and approximately $30 \mathrm{~min}$. for the WB-PET/CT exam. Immediately following the WB-PET/ CT exam the patients are taken to the PEM Flex system and imaged as follows: (1) craniocaudal (CC) view of the contralateral breast, (2) CC view of the ipsilateral breast, (3) medio-lateral oblique (MLO) view of the ipsilateral breast, and lastly (4) MLO view of the contralateral breast. Each acquisition is for 7 minutes and covers the full $24 \mathrm{~cm}$ wide FOV. In most cases a mild degree of breast compression is used to immobilize the breast. The action of compressing the breast tends to displace some tissue out of the FOV. In certain cases, especially when there is suspected involvement in the posterior breast, compression is greatly reduce or eliminated all together in order to image as far posterior as possible. 
Nearly 150 patients have been imaged with the PEM Flex Solo II at the Swedish Cancer Institute since July 2007. The PEM findings are currently being correlated with patient history and outcome, including comparison with mammography, ultrasound, WB-PET/CT, MRI, and pathological findings. In particular, PEM images are being correlated to MRI findings in the context of evaluating disease extent and guiding surgical treatment options. These findings are then compared against histological findings, which are considered the gold standard. Since this is an ongoing study we do not present any clinical results here. Examples of patient images are presented to illustrate representative clinical findings.

\section{Results}

\section{A. Spatial Resolution}

Figure 3 shows sample images of the capillary tube cross-sections, which were imaged in air. Both in-plane and cross-plane images of the point sources are shown. The images shown in figure 3 demonstrate the ability of the PEM Flex Solo II to distinguish I-mm point sources separated by $5 \mathrm{~mm}$. The images shown in figure 3 were not used to calculate spatial resolution, rather images with only one source, or sources separated by at least $10 \mathrm{~mm}$ were used for resolution calculations.

The mean spatial resolution measured at several locations within the FOV, and for three different compression thicknesses was $2.4 \pm 0.3 \mathrm{~mm}$ in the in-plane images, and $8.0 \pm 1.0 \mathrm{~mm}$ in the cross-planes.

\section{B. Uniformity}

The image of the uniform source that was acquired for 7 minutes is shown in figure 4 . The edge of the detectors are at the bottom of this image, and the source extends beyond the FOV there. Several profiles through each dimension of this image are shown in figure 5. The source, provided by a pair of IV bags, has rounded edges, so some variability at the source edge is expected. However, the interior of the source contains a uniform distribution of $18-\mathrm{F}$ concentration.

The ROIs used to calculate percent background variability (\%BV) are seen in figure 4 . Table 1 lists the \% BV for uniform images of different acquisition durations.

Theoretically the \%BV should decrease with increased acquisition duration due to the corresponding increase in counting statistics. The expected trend was not observed on these images, indicating that the within-image variability predominates the counting statistics variance.

The profiles (figure 5) show variation in counts across each dimension of the FOV. There is presently no attenuation or scatter correction in the reconstruction algorithm, which could be factors contributing to this variation. Also, the limited-angle sampling of the image is a potential contributor to non-uniformity.

\section{Edge Effects}

A plot of the true source position vs. the position in the PEM image is shown in figure 6 . The true source position was measured from the edge of the detector housing, against which the patient torso abuts. The image position was taken from an in-plane image, so each pixel is 1.2 $\mathrm{mm}$, thus the image position distance is the product of image pixel number from the edge of the image and the $1.2 \mathrm{~mm}$ pixel size. Figure 6 shows that the accurate active imaging area of the PEM Flex Solo II begins approximately $13 \mathrm{~mm}$ from the edge of the detector housing. 
The coefficient of variation ( $\mathrm{CV}=$ standard deviation / mean) was calculated for entire crossplanes near the FOV edge, and for image profiles near the edge of in-plane images. Figure 7 shows graphs of each of these as a function of distance from the edge of the FOV. CV increases at the FOV edge as expected due to the limited coincidence sampling there. This effect leads to noticeable edge artifacts of varying degrees on the PEM Flex images. As mentioned earlier, the methods used in WB-PET for mitigating reduced edge sensitivity are not possible on the PEM Flex Solo II. Consequently, a trade-off must be made between accepting edge artifacts due to reduced counting statistics, and eliminating these edge slices from the image at the cost of reduced posterior imaging capability. We discuss this trade-off below from the perspective of clinical image evaluation in the patient examples.

\section{Recovery Coefficients}

Figure 8 shows an image acquired with four of the spheres used in RC experiments. This image illustrates visualization of various size spheres with $4 \mathrm{X}$ the background activity concentration. Figure 8 indicates that a $5 \mathrm{~mm}$ diameter sphere is about the limit for seeing lesions $4 \mathrm{X}$ the background activity. This image was not used to calculate RC; we used images with spheres near the FOV center for those calculations.

Figure 9 shows the RC values plotted against sphere diameter. As expected, the measured RC values approach the true activity concentration ratio for larger sphere sizes. Of note on the RC plot is the fact that the $\mathrm{RC}$ values are different when the spheres are centered between the detectors, or placed on one of the detectors; the 'on-detector' RC curves appears to level out just above the true ratio value, whereas the center FOV RC curves level out just below the true ratio value.

Imaging the $2 \mathrm{~cm}$ diameter sphere at the four cardinal locations within the FOV yielded the relative activity concentration values shown in figure 10. As with the RC measurements, the activity concentration was found to be greater when the source was positioned on the detectors than when centered between the detectors. There is also a trend towards higher measured activity at the posterior FOV edge (i.e. near the patient) relative to the center. While the background activity was present for these experiments, the relative values reported here are from ROIs drawn entirely inside the sphere; background activity did not figure into the calculation.

\section{E. Clinical Examples}

Results of the clinical PEM finding are currently being accumulated and tabulated at the Swedish Cancer Institute. The patient images presented here represent interesting cases and consider issues relevant to PEM imaging technology and technique.

Fig. 11 shows a case indicative of the application for which PEM is being used at the Swedish Cancer Institute. In this case, a patient with a known primary tumor in the right breast was imaged with the PEM Flex. The primary tumor is large with high FDG uptake. Not evident on the WB-PET/CT image is a small second lesion with uptake significantly above background. Also visualized is an area of moderate FDG uptake following ductal patterns, making it suspicious for ductal carcinoma in situ (DCIS). These findings were supported by MRI, and confirmed on pathologic examination. This is a case in which surgical treatment was guided by the finding of the secondary malignant lesion, the multi-focal nature of the disease likely eliminates this patient as a candidate for breast-conservation surgery.

Figure 12 shows a case where a lesion with high FDG uptake is located in a posterior position. The lesion appears on the edge of the $\mathrm{CC}$ view images, and just slightly off the edge of the MLO views. This suggests that MLO views can image more posterior tissue. In this case the 
lesion is sufficiently large and sufficiently radioactive that it is easily distinguished from the edge-artifacts in the images.

Figure 13 shows a case similar to that above, except in this patient the posterior lesion was seen only on the MLO view, and not on the CC view. Again, this lesion is hot enough and large enough to be distinguished from edge artifacts. However, the accuracy of localizing this lesion in the M-L dimension suffers without visualization provided by the orthogonal CC view.

Finally, figure 14 shows an image with indeterminate FDG uptake. This finding is in the contralateral breast; a known primary tumor was seen by WB-PET/CT, PEM, and other means in the left breast. Figure 14 shows the PEM image of the right breast, in which a focal hot spot is clearly seen. However, this lesion was not identified, even retrospectively, on other imaging modalities.

This case demonstrates the need for a tissue sampling mechanism associated with PEM systems. Suspicious findings on PEM may be candidates for biopsy, and if other imaging modalities cannot localize the suspicion, then biopsy can only be guided by PEM. Naviscan is reportedly going through FDA approval of a biopsy-guidance system for the PEM Flex Solo II system. In the meantime, these types of findings are followed more closely than non-findings (e.g. six-month follow-up rather than 12 month). In the particular case shown in figure 14 the patient opted for prophylactic mastectomy, which allowed a pathological examination of the suspected tissue. While there were a number of atypical findings, no malignant cancer was found, or other known indications of elevated FDG uptake. Benign FDG findings in the breast include uptake in fat necrosis, and near the nipple. The uptake in figure 14 was not fat necrosis, so this was an indeterminate false positive.

\section{Discussion}

We performed several experiments to characterize a commercial positron emission mammography scanner - the PEM Flex Solo II (Naviscan, Inc.). Since there is no standard for testing dedicated breast PET or PEM systems, we selected performance parameters that could be easily measured with the standard clinical software on the PEM Flex, and that were relevant to the breast imaging application. All analyses were done on images reconstructed using the default 3D list-mode MLEM program on the PEM scanner. The system has also been tested separately using the NEMA NU 4-2008 Small Animal PET standard [5].

Our results showed a $2.4 \pm 0.3 \mathrm{~mm}$ FWHM spatial resolution for in-plane image slices, in agreement with [5]. We measured $8.0 \pm 1.0 \mathrm{~mm}$ FWHM in the cross-plane images, slightly greater than in [5] (7 mm FWHM). Cross-plane images have worse resolution because this is a limited-angle imaging system (tomosynthesis). Cross-plane images are not intended for viewing.

The percent background variability was measured between 5-7\% depending on acquisition time. This variation is believed to be due to systematic non-uniformities in the image, since the $\% \mathrm{BV}$ did not vary with counting statistics as expected. The default image reconstruction does not include corrections for attenuated or scattered photons, which is a likely contributor to observed non-uniformities. Limited-angle sampling may also contribute.

The coefficient of variation in the PEM images increases at the edge of the FOV due to limited coincidence-count sampling at these positions (fig. 7). There is a trade-off between extending the FOV and limiting image variance at the edge: using all image planes that the PEM detector can measure gives the maximum FOV, but results in high variance at the FOV edge. This effect is seen as artifacts at the edges of the PEM Flex images (e.g. fig. 8, 11-14). However, as illustrated in figs. 12 and 13, maximizing the FOV can be important for patient imaging, despite 
the image artifacts at the edge. In the examples in figs. 12 and 13 the lesions were large enough and hot enough to be easily distinguished from the variance artifacts. Had these image slices been omitted for the sake of low overall image variance, then those lesions may not have been visualized. Maximizing the FOV is therefore important, with proper care at interpreting the edge of the images. Cases in which lesions are smaller and less active, such that they cannot be distinguished from the variance artifacts, must be read as inconclusive. This is an area for further consideration concerning the design, evaluation, and implementation of dedicated breast PET and PEM systems.

We performed a simple test of the shielding at the edge of the PEM Flex detectors to investigate whether any of the spurious edge counts contributing to the artifacts there were due to random coincidence events from outside the FOV. The relative number of random coincidence events did not change appreciably when we placed additional shielding between the detector and the source outside the FOV. Contamination from outside FOV activity is another effect that should be included in standardized testing of dedicated breast PET and PEM systems.

The PEM Flex Solo II provides high-resolution and dedicated breast images that show greater tracer uptake detail that what can be seen with standard WB-PET scanner protocols. The highresolution is well suited to identify multi-focal and multi-centric disease, which is an important consideration for treatment planning. PEM Flex image quantification was variable within the FOV (fig. 9, 10), making absolute quantitation measurements challenging from a reproducibility standpoint. Adding attenuation and scatter correction should improve quantification, yet fundamental limitations may persist due to the limited-angle sampling used to generate images.

As interest in dedicated breast PET and PEM increases, and more systems are developed and placed in clinics, a standard method for comparing and characterizing systems must be developed. As was done for small animal PET scanners, a modified form of the WB-PET testing standard should be developed that takes into account the size and configuration of dedicated breast scanners, and includes tests specifically relevant to breast imaging, such as edge detection and random coincidences from outside FOV.

\section{Acknowledgments}

The authors thank Chris Matthews of Naviscan Inc. for useful discussions in this work, and Jennifer Coburn, Joiem Kawas, and Kris Kohn of the Swedish Cancer Institute for their assistance with experiments.

This work was supported in part by NIH Grant numbers 5ROICA074135 and 5ROICA115870.

\section{References}

[1]. Elmore JG, Annstrong K, Lehman CD, Fletcher SW. Screening for breast cancer. JAMA 2005;293 (10):1245-1256. [PubMed: 15755947]

[2]. Eubank WB, Mankoff DA. Current and future uses of positron emission tomography in breast cancer imaging. Semin Nucl Med 2004;34:224-240. [PubMed: 15202103]

[3]. Thompson CJ, Murthy K, Weinberg IN, Mako F. Feasibility of positron emission mammography. Med Phys 1994;21:529-538. [PubMed: 8058019]

[4]. Thompson CJ. fustrumentation for positron emission mammography. PET Clin 2006;1:33-38.

[5]. Luo, W.; Yarnall, S.; Anashkin, E.; Matthews, CG. First Test Results of a Commercially-Available Clinical PET Scanner Using the NEMA NU 4-2008 Small Animal PET Standard; Conference Proceedings, 2008 NSS-MIC; Dresden, Germany. 2008; Oct. 19-25.

[6]. NEMA. "Performance measurements of positron emission tomographs". Nat. Elect. Manufact. Assoc.; Rosslyn, VA: 2001. NEMA Standards Pub. NU 2-2001 


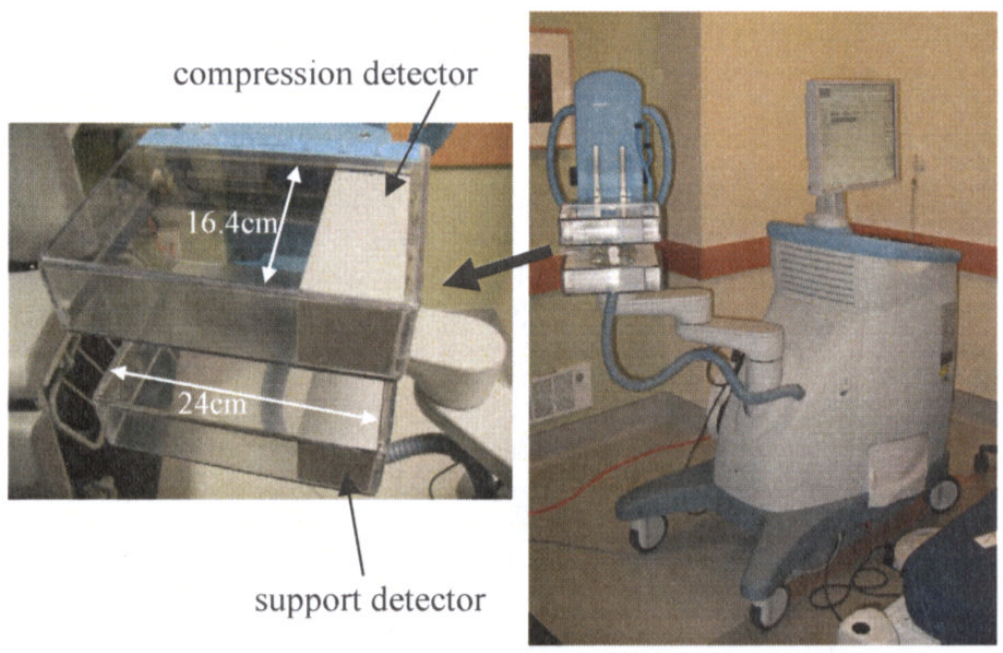

Fig. 1.

Photographs of the PEM Flex Solo II. RIGHT: the full system; acquisition console, articulating ann, and detector paddles. LEFT: close-up of the detectors in their compression paddle housing. The clear plastic housing immobilizes the breast while the detectors scan in unison inside the housing paddles. 


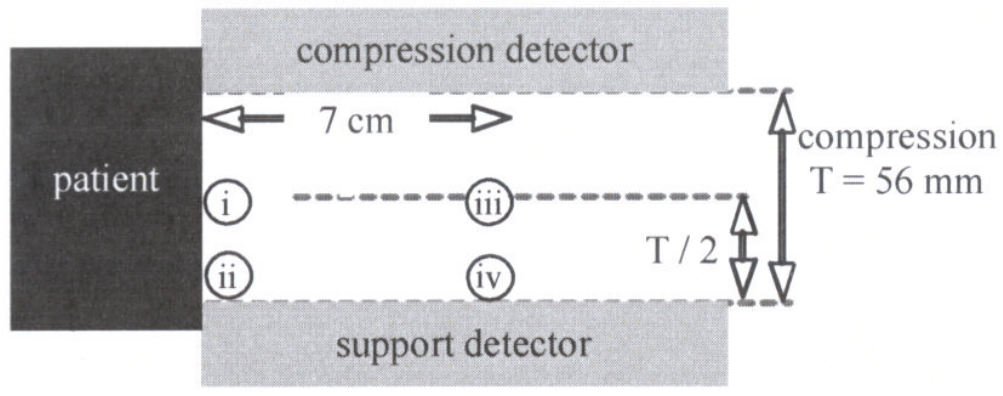

Fig. 2.

The four cardinal positions within the PEM Flex FOV used to investigate quantification consistency: (i) far posterior (near patient) centered between detectors, (ii) far posterior in contact with a detector, (iii) center of entire FOV, and (iv) anterior-posterior center, in contact with a detector. The detectors scan perpendicular to the plane of the figure; edges of the FOV in the scan direction were not tested. 

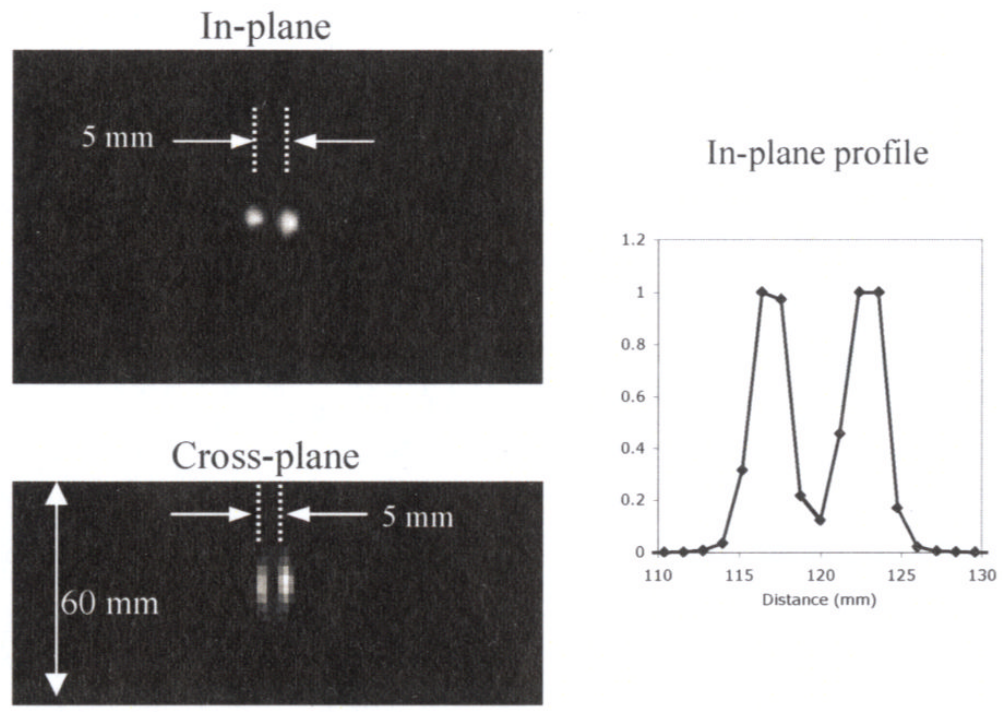

Fig. 3.

In-plane and cross-plane images of two I-mm point sources (in air) separated by $5 \mathrm{~mm}$. Note spatial anisotropy of the cross-plane (the in-plane image pixels and resolution are isotropic). Profile through the in-plane image shows a 10-to-1 peak-to-valley ratio, and also illustrates how spatial resolution is limited by image pixel size in these images. 


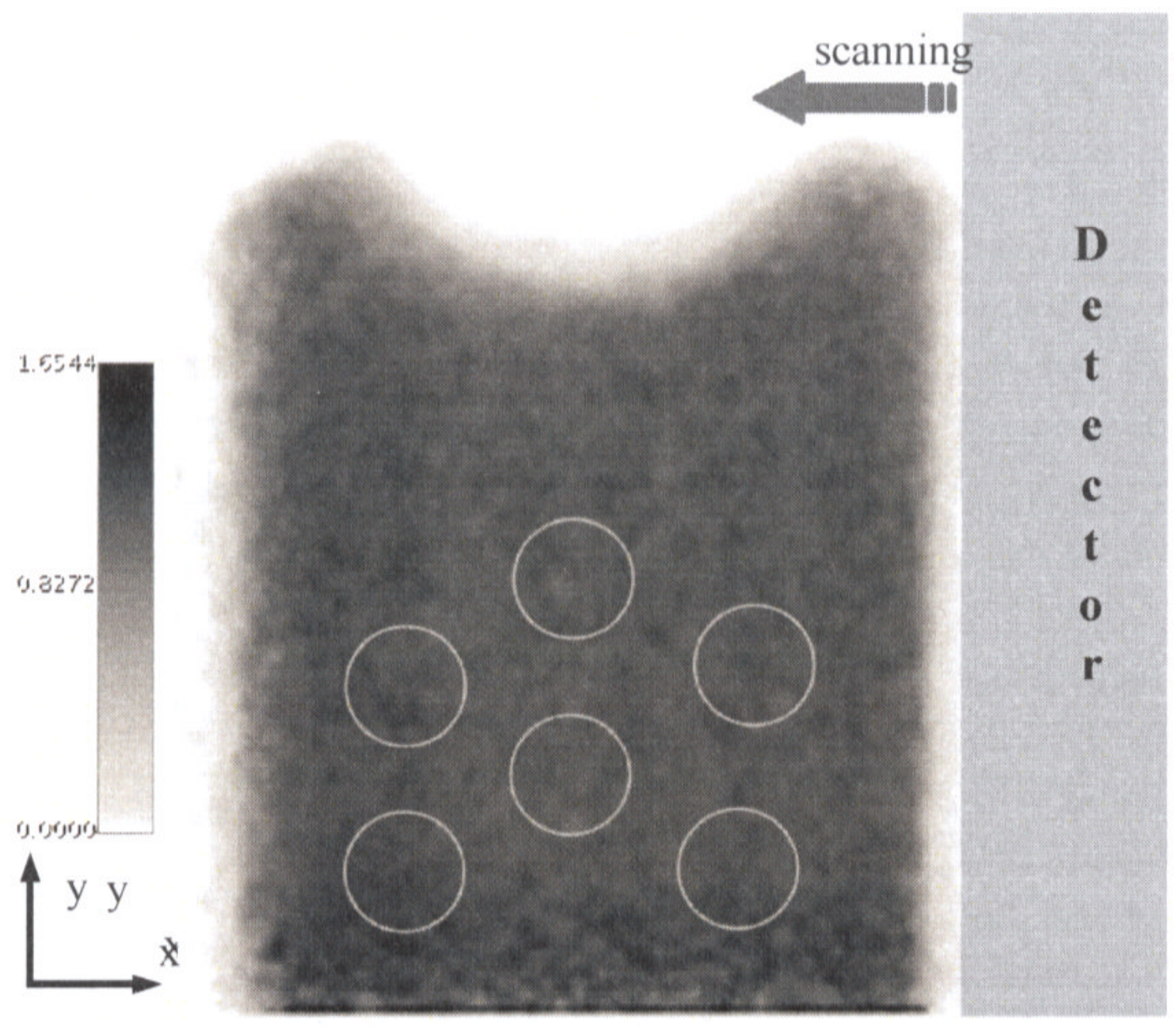

Figure 4.

Image of uniform source: in-plane slice 13 of 24 . Gray circles are the $2 \mathrm{~cm}$ diameter ROIs used to calculate \%BV. Six such ROIs are drawn on each image slice. The $\mathrm{x}$-axis is the scan direction of the detectors. The y-axis is along the length of the detectors. 

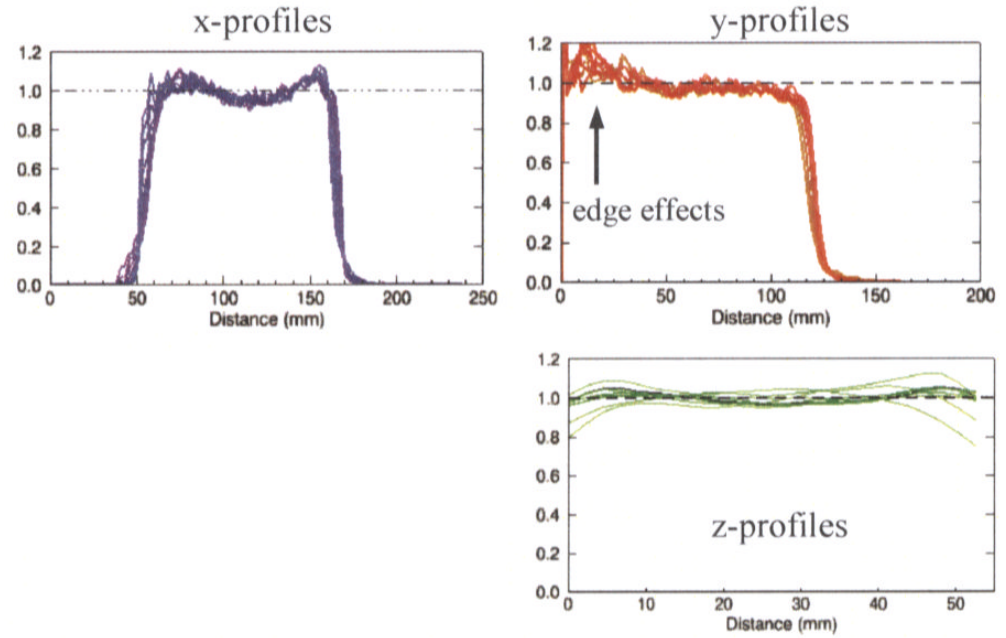

Fig. 5.

Profiles through the uniformity image. Each profile is the sum of 10 central adjacent rows or columns. Profiles from several image slices are shown. Axes are defined in Fig. 4. 


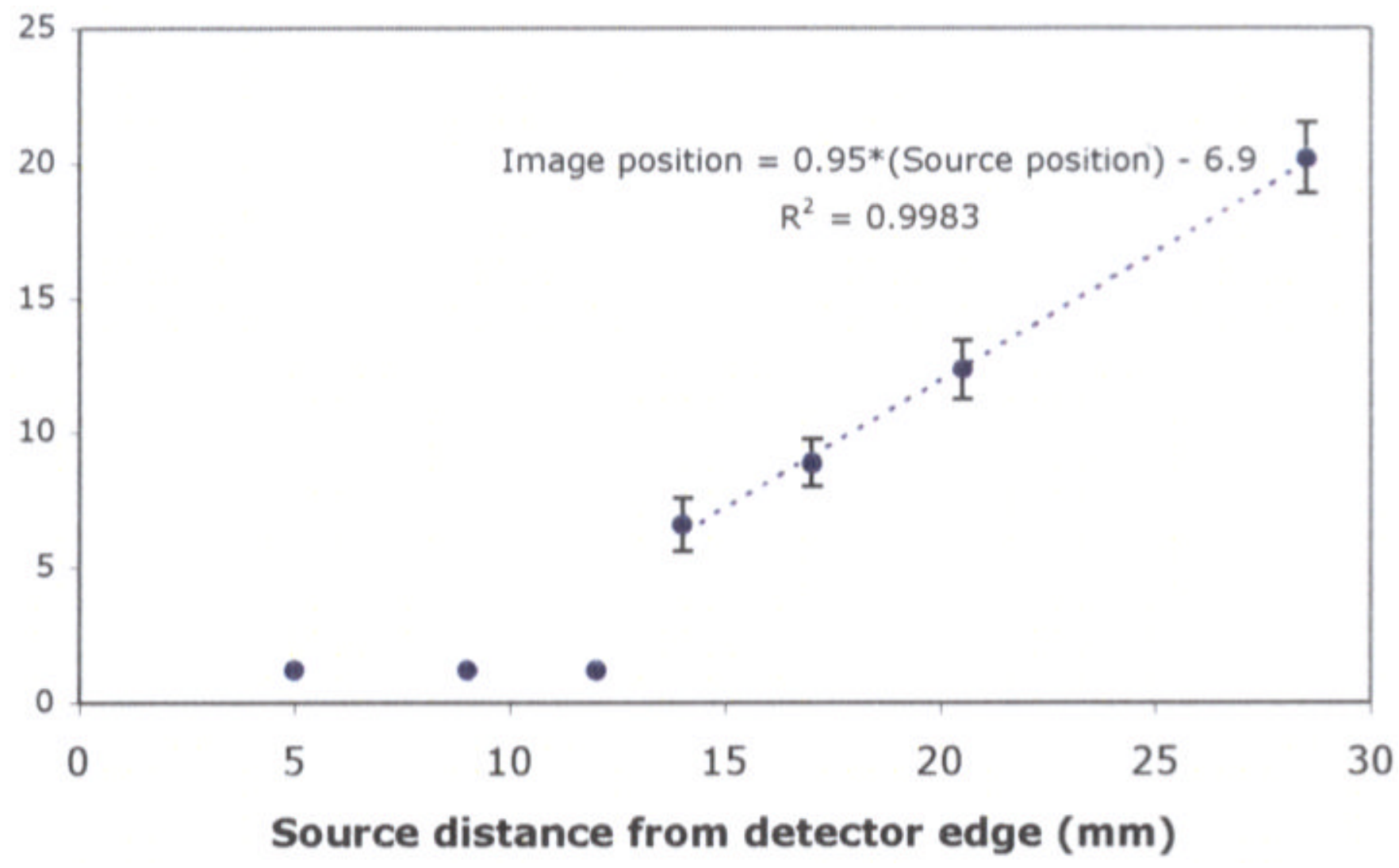

Fig. 6.

A line source was stepped from the edge of the detector housing (source distance $=0 \mathrm{~cm}$ ) towards the FOV center. This graph shows the resulting source position measured on the corresponding images. 

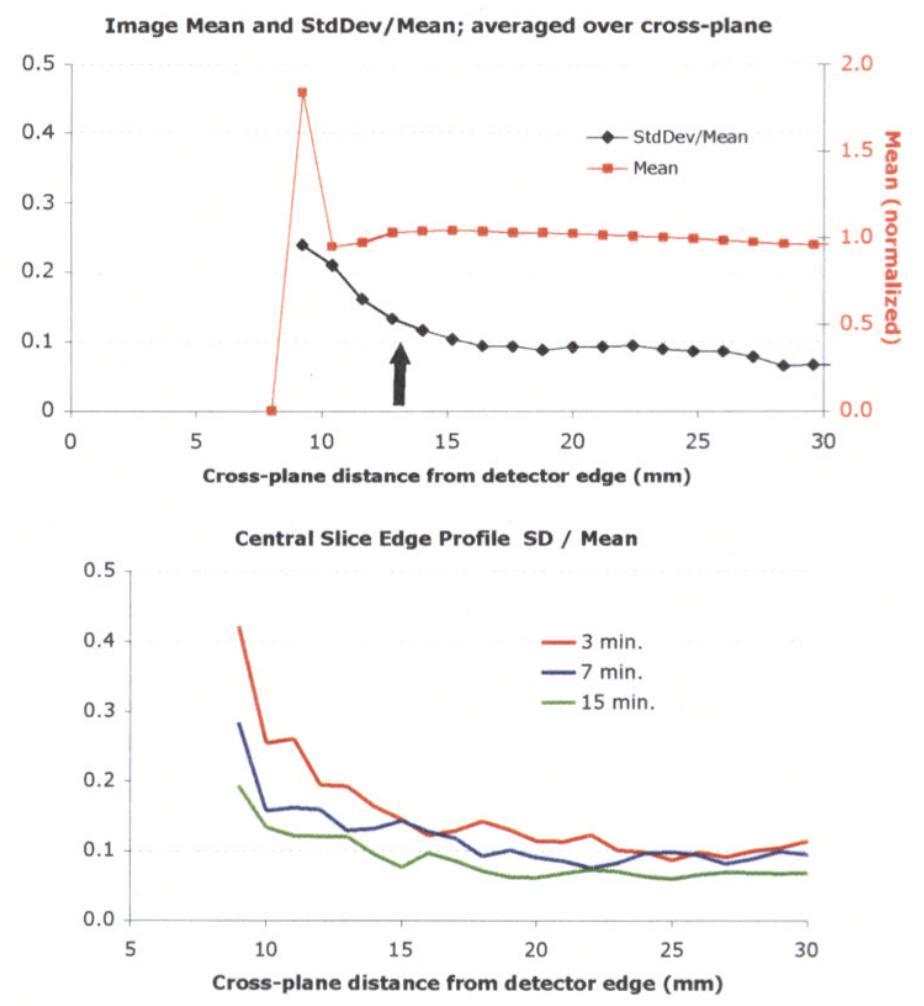

Figure 7.

TOP: The mean and CV of 2-D ROIs in the cross-planes vs. distance from the detector edge. Arrow indicates where accurate imaging begins (determined from fig. 6) BOTTOM: CV of ID profiles from central in-plane image. Results are shown for image acquisitions of 3, 7, and 15 min. Distances correspond to those shown in fig. 6. 


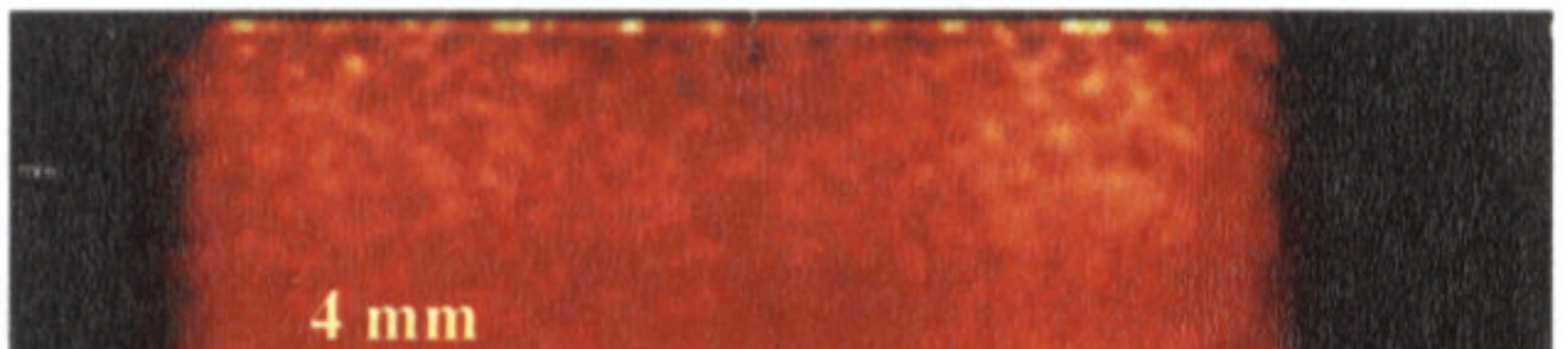

$5 \mathrm{~mm}$

$8 \mathrm{~mm}$

$20 \mathrm{~mm}$

Fig. 8.

Image of four spheres positioned between the two IV-bag background sources. The sphere inner diameter is specified on the image. 


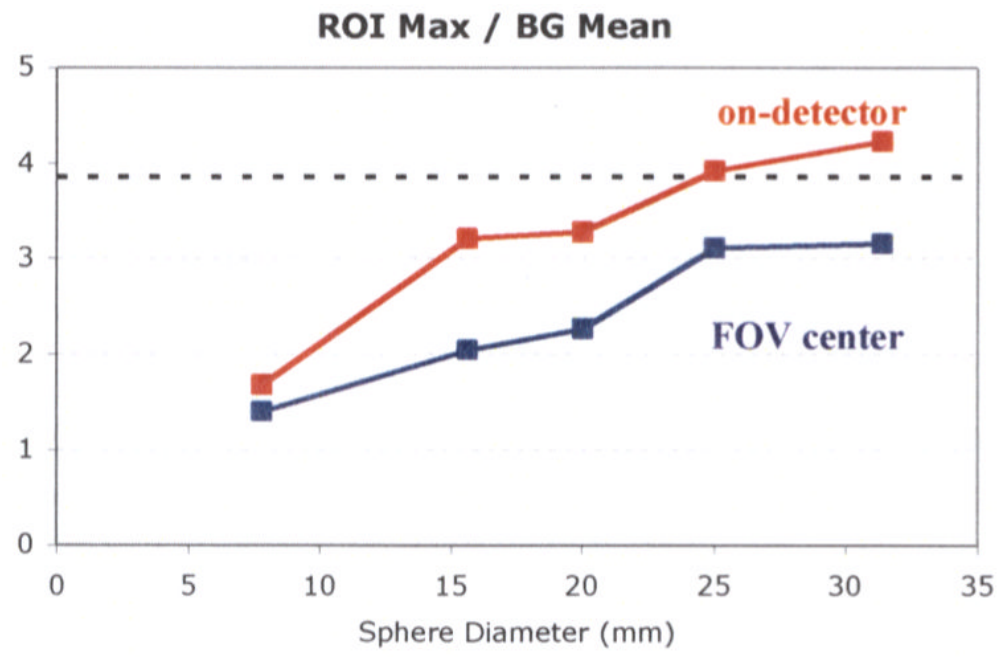

Fig. 9.

Recovery coefficients vs. sphere diameter. 


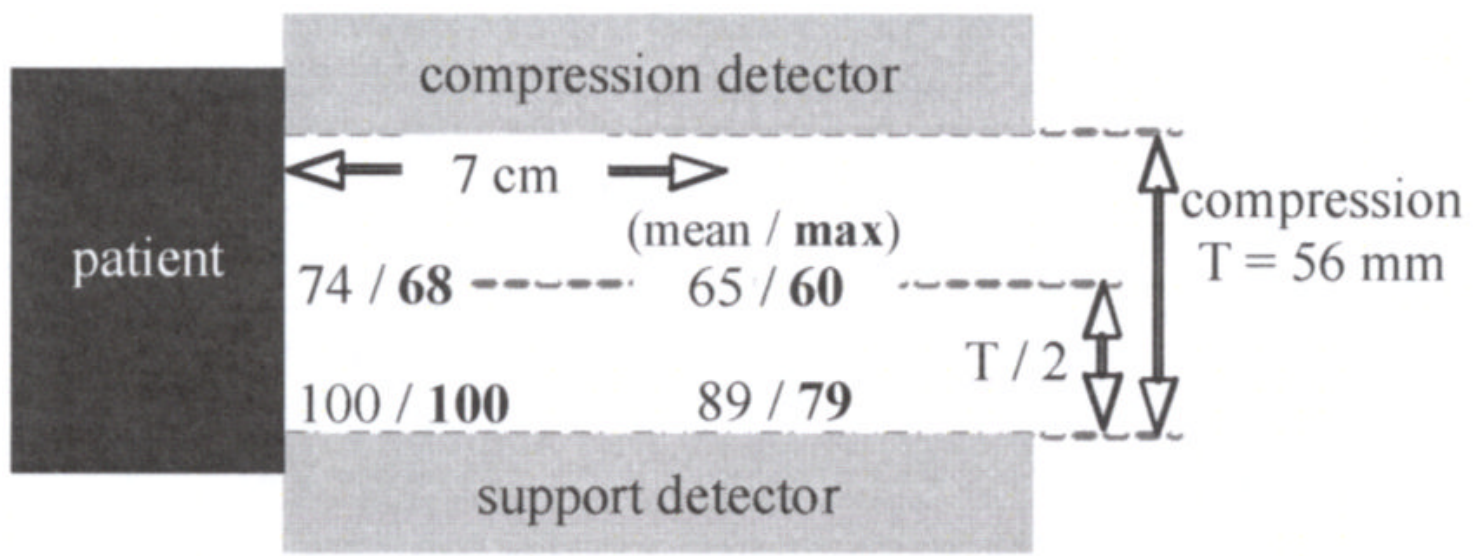

Fig. 10.

Relative mean and relative maximum activity concentrations (decay corrected) measured from a single source imaged at four unique positions within the FOV. The mean and maximum (mean / max) values are given as normalized to the highest respective mean or max value. The highest value was in the lower posterior position for both mean and max. The greater relative variation in the max values is not unexpected. 


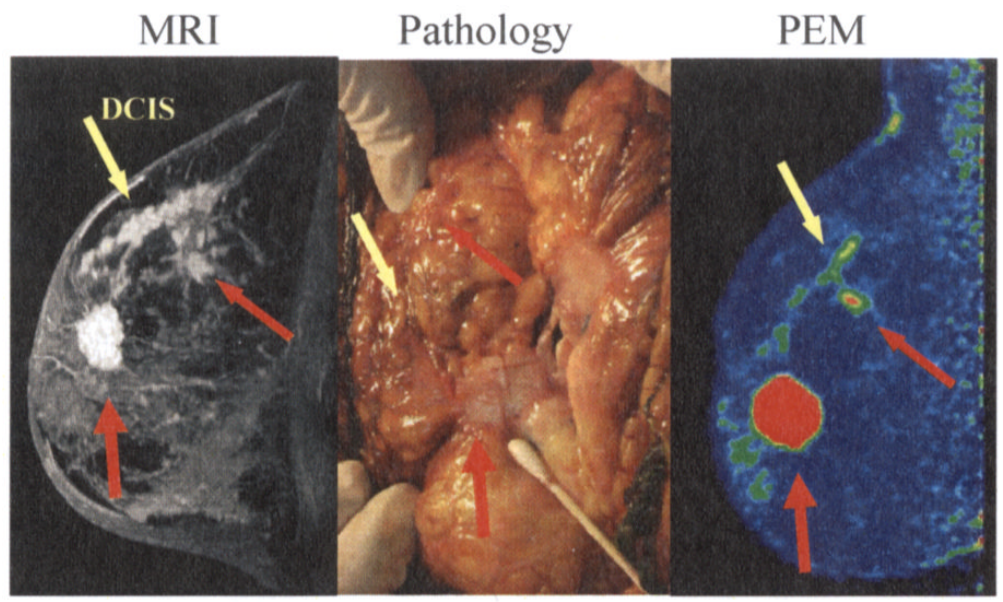

Fig. 11.

LEFT: MRI indicated primary and secondary lesions (red arrows) and pre-cancerous DCIS (yellow arrow). MIDDLE: Pathology confirmed imaging findings. RIGHT: PEM image also shows secondary lesion and DCIS. 
CC

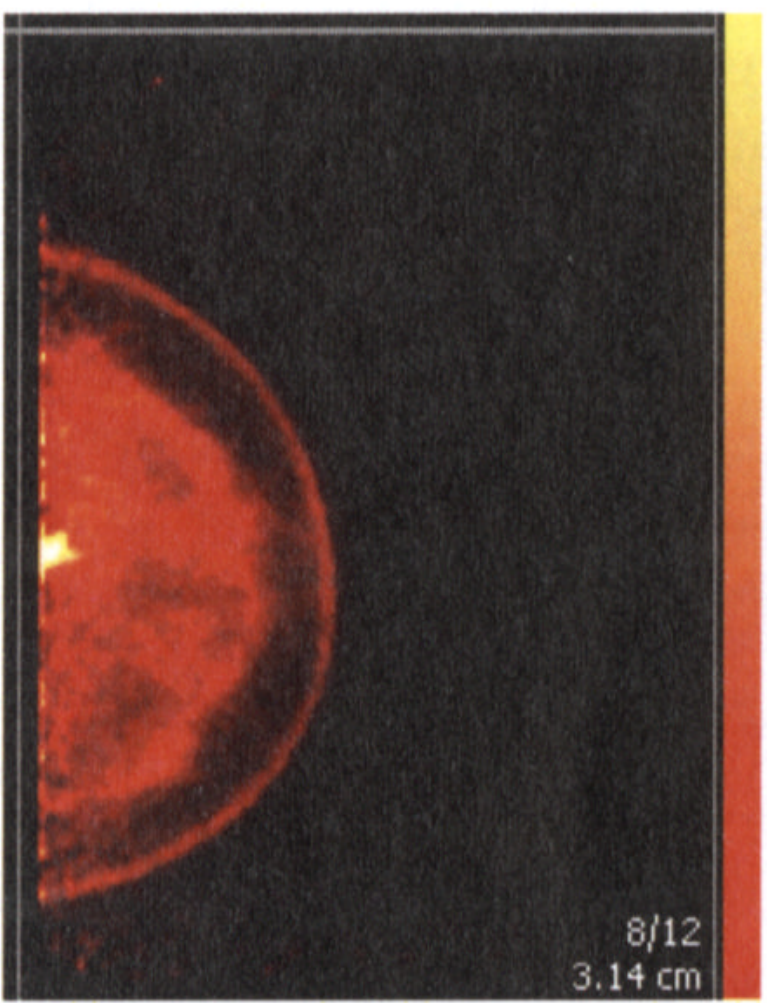

MLO

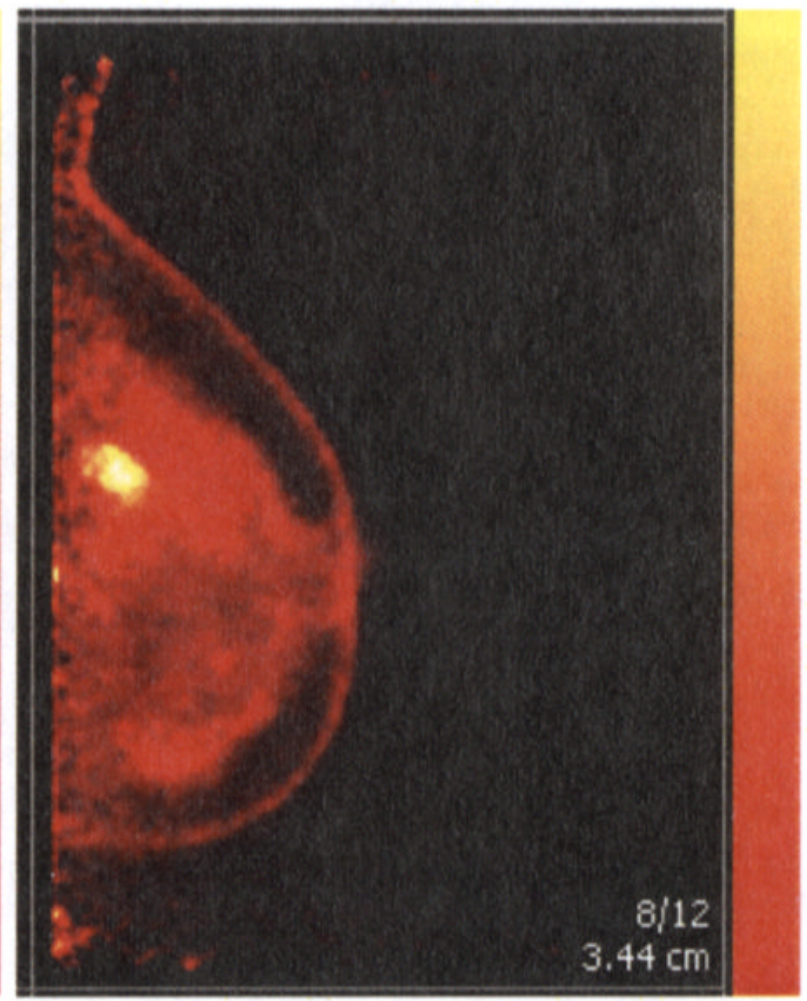

Fig. 12.

Example of a lesion on the FOV edge in the CC view, and just off the edge in the MLO view. 


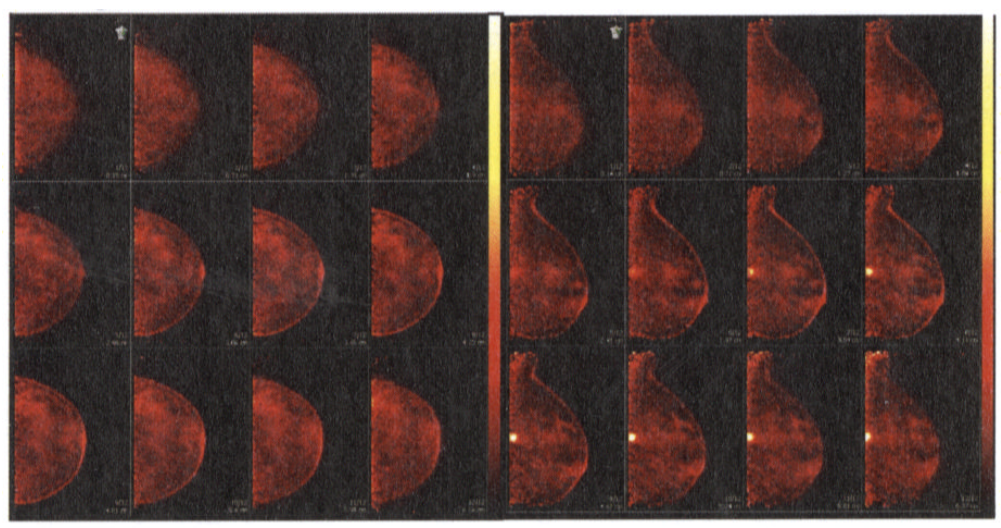

Fig. 13.

Lesion at the FOV edge is seen on MLO, but not on CC view. 

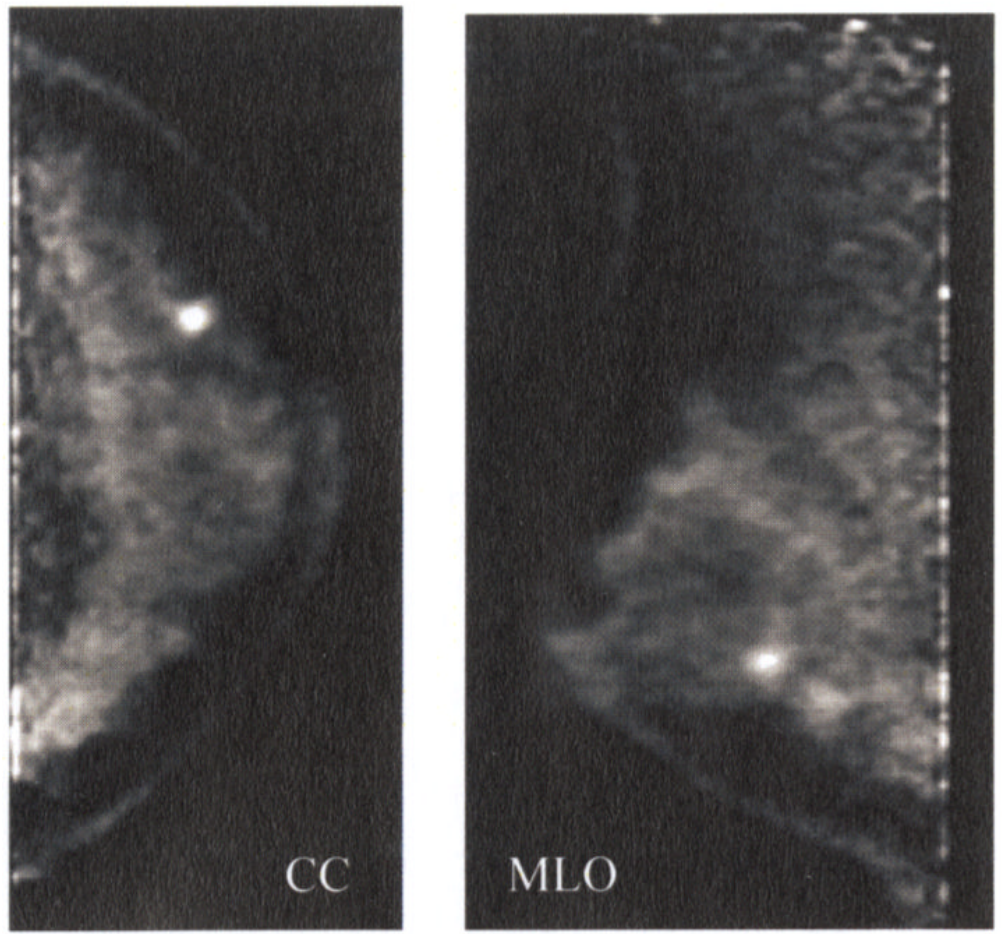

Fig. 14.

CC and MLO views of an indetenninate FDG uptake focus. 


\section{Table 1}

\%Background Variability

\begin{tabular}{cc}
\hline Acg.Duration (min.) & \% BV \\
\hline 1 & 5.1 \\
3 & 5.6 \\
7 & 7.0 \\
15 & 6.0 \\
\hline
\end{tabular}

